\title{
Role of van der Waals interaction in forming molecule-metal junctions: flat organic molecules on the $\mathrm{Au}(111)$ surface $\dagger$
}

\author{
M. Mura, ${ }^{a}$ A. Gulans, ${ }^{b}$ T. Thonhauser ${ }^{c}$ and L. Kantorovich ${ }^{* a}$ \\ Received 29th September 2009, Accepted 12th February 2010 \\ First published as an Advance Article on the web 17th March 2010 \\ DOI: $10.1039 / \mathbf{b 9 2 0 1 2 1 a}$
}

The self-assembly of flat organic molecules on metal surfaces is controlled, apart from the kinetic factors, by the interplay between the molecule-molecule and molecule-surface interactions. These are typically calculated using standard density functional theory within the generalized gradient approximation, which significantly underestimates nonlocal correlations, i.e. van der Waals (vdW) contributions, and thus affects interactions between molecules and the metal surface in the junction. In this paper we address this question systematically for the $\mathrm{Au}(111)$ surface and a number of popular flat organic molecules which form directional hydrogen bonds with each other. This is done using the recently developed first-principles vdW-DF method which takes into account the nonlocal nature of electron correlation [M. Dion et al., Phys. Rev. Lett. 2004, 92, 246401]. We report here a systematic study of such systems involving completely self-consistent vdW-DF calculations with full geometry relaxation. We find that the hydrogen bonding between the molecules is only insignificantly affected by the vdW contribution, both in the gas phase and on the gold surface. However, the adsorption energies of these molecules on the surface increase dramatically as compared with the ordinary density functional (within the generalized gradient approximation, GGA) calculations, in agreement with available experimental data and previous calculations performed within approximate or semiempirical models, and this is entirely due to the $\mathrm{vdW}$ contribution which provides the main binding mechanism. We also stress the importance of self-consistency in calculating the binding energy by the vdW-DF method since the results of non-self-consistent calculations in some cases may be off by up to $20 \%$. Our calculations still support the usually made assumption of the molecule-surface interaction changing little laterally suggesting that single molecules and their small clusters should be quite mobile at room temperature on the surface. These findings support a gas-phase modeling for some flat metal surfaces, such as $\mathrm{Au}(111)$, and flat molecules, at least as a first approximation.

\section{Introduction}

Metal-organic interfaces ${ }^{1-3}$ have been attracting increasing interest recently, both experimentally ${ }^{4-17}$ and theoretically $y^{6,8-12,15,18-30}$ due to the growing number of their applications in nanotechnology, specifically, as a tool for constructing porous templates for incorporation of "guest" molecules to achieve the desired functionalization ${ }^{3,24,31-39}$ and for building nanodevices ${ }^{40,41}$ to name just a few.

Particular structures formed during the deposition depend crucially on the interplay between the molecule-molecule and molecule-surface interactions which determine the kinetics steering the "construction" process. ${ }^{42-44}$ If the moleculemolecule interaction dominates, then molecules may move

\footnotetext{
${ }^{a}$ Physics, King's College London, The Strand, WC2R 2LS, London,

UK.E-mail:lev.kantorovitch@kcl.ac.uk

${ }^{b}$ COMP/Department of Applied Physics,

Aalto University School of Science and Technology,

P.O. Box 11100, FI-00076 AALTO, Finland

${ }^{c}$ Department of Physics, Wake Forest University, Winston-Salem, NC 27109, USA

$\dagger$ Electronic supplementary information (ESI) available: Further information on the diffusion calculations of the melamine and PTCDA molecules on the $\mathrm{Au}(111)$ surface. See DOI: 10.1039/b920121a
}

around the surface to form molecular assemblies (providing, of course, that the surface diffusion is not hampered by a too large deposition rate $^{2,31,32}$ ); in this case commensurability issues do not arise and the gas-phase modeling of the assemblies, ${ }^{7-9,11,27}$ i.e. without considering the surface, is justified. If, however, the molecule-surface interaction is much stronger than their mutual interaction, then all depends on the actual energy barriers for the surface diffusion: in the case of small barriers, the molecules can still move around to form the assemblies steered by inter-molecular interactions; if the barriers are significant, then one has to start the analysis by finding all possible adsorption sites. In the intermediate case the full calculation involving the assembly and the surface should be performed and the whole potential energy surface analyzed, i.e. in this case the commensurability analysis becomes essential.

Therefore, the calculation of binding energies of the molecules with the surface, energy barriers for their diffusion as well as of interaction energies between the molecules for realistic molecule-metal junctions is of crucial importance. However, up to now the dispersion interaction has not been properly included when considering these types of interactions. 


\section{Theoretical background and motivation}

Density Functional Theory (DFT) has become an invaluable tool in studying molecular assemblies (see, e.g. ref. 6,8-12,15 and 18-29). However, in many cases, e.g. for DNA bases $^{8,11,12,14,16}$ and melamine molecules on the $\mathrm{Au}(111)$ surface, ${ }^{15,28} \quad$ perylene-3,4,9,10-tetracarboxylic-3,4,9,10dianhydride (PTCDA) molecules on the $\operatorname{Ag}(111)$ surface, ${ }^{19,22,23}$ benzene $^{26}$ and PTCDA ${ }^{30}$ molecules on the $\mathrm{Au}(111), \mathrm{Cu}(111)$ and $\operatorname{Ag}(111)$ surfaces the calculated adsorption energies are too small (of the order of $0.1-0.2 \mathrm{eV}$ ) which is inconsistent with experimental observations of the desorption temperatures for these molecules (around 100-300 ${ }^{\circ} \mathrm{C}$ ). This failure of standard generalized gradient approximation (GGA) functionals, such as e.g. Perdew-Burke-Ernzerhof (PBE) ${ }^{45}$ is attributed to incorrect description of the electron correlation in situations when the electronic densities of the molecule and surface practically do not overlap and the dispersion interaction, or van der Waals (vdW) forces, become dominant. ${ }^{26,46}$ Note that the local density approximation (LDA) typically predicts stronger binding than GGA, also for covalent systems, however, this effect is artificial. ${ }^{47,48}$

There have been attempts to rectify the problem by supplementing DFT calculations with additional semiempirical terms which take into account dispersion interactions (DFT-D method). ${ }^{49}$ Because of the parameterization, however, this approach is of limited flexibility. Indeed, by construction, semiempirical approaches are bound to work for systems they were designed for; strictly speaking, one never can be sure if the results obtained with such theories are reasonable for systems which are outside that set used for parameterization. Only methods which are free from it have an ability to perform uniformly across a wide range of different systems.

Recently a truly first-principles approach, which does not require any fitting parameters and still stays within the remit of DFT (the total energy of electrons is a unique functional of the electron density $\rho(\mathbf{r}))$, was developed ${ }^{50-52}$ and successfully applied to many systems. ${ }^{48}$ In this method the energy functional has the form:

$$
E_{\mathrm{vdW}-\mathrm{DF}}[\rho]=E_{\mathrm{GGA}}[\rho]-E_{\mathrm{GGA}, \mathrm{c}}[\rho]+E_{\mathrm{LDA}, \mathrm{c}}[\rho]+E_{\mathrm{c}}^{\mathrm{n}}[\rho]
$$

where $E_{\mathrm{GGA}}$ is the total energy corresponding to the density functional of the chosen GGA flavor and the other three terms provide a correction to it. $E_{\mathrm{c}}^{\mathrm{nl}}$ represents a truly non-local contribution to the correlation energy and accounts for the dispersion interaction, while the difference of the correlation energies, $E_{\mathrm{LDA}, c}[\rho]-E_{\mathrm{GGA}, \mathrm{c}}[\rho]$, serves to replace the correlation energy contained in the GGA functional with the truly local contribution of LDA. This is done to avoid any possible double-counting of the non-local contributions to the correlation energy. It is argued in ref. 50-52 that the revPBE density functional ${ }^{53}$ is the best choice for the GGA flavor to be used in the vdW-DF since it predicts negligible or no binding in vdW complexes due to the exchange alone.

Until recently the last term $E_{\mathrm{c}}^{\mathrm{nl}}$ was the most challenging in practical implementations of the method as it involves a double spatial integral. It required a significant computational effort and as a result in most calculations employing this density functional ${ }^{30,46,50-52,54-56}$ the correction to GGA was added a posteriori. Such a non-self-consistent approach, although approximate, may work in many cases when the correction depends weakly on the density and only mildly rearranges it. However, it is difficult to verify the validity of such an approximation for a specific problem. Since the calculation of atomic forces was also not available with the new functional, usually atomic geometries were obtained using the chosen GGA functional prior to the vdW correction to be applied, or geometry relaxation was performed with respect to a limited number of degrees of freedom using the total energy.

These limitations have been recently overcome $e^{57-59}$ and the fully self-consistent vdW-DF calculations with complete geometry relaxation can now be performed routinely on systems consisting of hundreds of atoms. Here for the first time we report such calculations on a number of flat organic molecules adsorbed on the $\mathrm{Au}(111)$ surface with the aim of guiding the theoretical effort required to investigate supramolecular assemblies on flat metal surfaces. These recent developments represent a remarkable achievement as calculations of this kind and scale were not possible before. Our calculations here are based on a new implementation of the vdW-DF ${ }^{57}$ in the SIESTA code. ${ }^{60}$ The numerical approach is based on an adaptive quadrature real-space grid which allows an efficient calculation of both the Kohn-Sham potential and the total energy. As a result, fully self-consistent calculations of energies and forces became available at an additional cost which is just a fraction of the cost of the usual GGA calculation.

Our main concern is to understand the role played by the $\mathrm{vdW}$ interaction in formations of hydrogen bonded assemblies of simple flat molecules on this surface. The $\mathrm{Au}(111)$ surface was chosen since it represents a popular platform for looking for flat ordered molecular arrangements: it can be easily prepared with relatively large terraces available, is well studied, and allows performing Scanning Tunneling Microscopy (STM) experiments. The main questions we ask here are: (i) what is the main binding mechanism of the molecules to stick to the surface and what are the binding energies; (ii) does the binding to the surface depend on the size of the molecules; (iii) how is the kinetics of the molecules on the surface affected by the vdW interaction; (iv) how does the vdW interaction affect the hydrogen bonding interaction both in the gas phase and on the surface, and, finally, (v) in view of many calculations performed so far $^{30,46,50-52,54-56}$ when the vdW correction was added a posteriori, we also ask how important is electronic self-consistency within the vdW-DF method. The first and the third questions have been addressed in ref. 11, 12, 15 and 28 for some DNA bases on the gold surface using a semiempirical approach based on the correction for the vdW interaction obtained in ref. 61 by fitting to second order Møller-Plesset (MP2) perturbation calculations of gold clusters with fragments of nucleic base molecules. It was found that although binding (adsorption) energies increase substantially due to the dispersion interaction, the lateral molecule-surface potential remains rather flat as given by the GGA calculations. Here for the first time we perform fully first-principles calculations which basically confirm these findings. Concerning the other questions above, some of the 
hydrogen-bonding systems have already been studied previously using the vdW-DF method. ${ }^{56,57}$ Here we add to the discussion by studying a different set of systems using both PBE and vdW-DF. All our calculations are fully self-consistent and the complete geometry relaxation was consistently performed in all cases. We also compare a hydrogen bonded system in the gas phase and on the surface and check their relative energies, and fully self-consistent calculations are compared with non-self-consistent ones.

\section{Computational details}

For all our calculations we used a modified version of SIESTA, which is based on periodic boundary conditions (PBC), localized basis set and the method of pseudopotentials. ${ }^{60}$ We used a double zeta polarized (DZP) basis set for all species with the confinement regions corresponding to the energy shift of $10 \mathrm{meV}$. All vdW-DF calculations were performed using revPBE as the GGA functional in eqn (1), and these are compared here with the results obtained using the GGA method based on the PBE density functional. All structures considered were completely optimized with the force tolerance of $0.01 \mathrm{eV} \AA^{-1}$. To calculate the binding (or stabilisation) energies, the counterpoise correction $^{62}$ for the basis set superposition error (BSSE) has been applied in all cases. Most of our calculations have been done using the 250 Ry plane wave cutoff for the grid.

The binding energies of dimers of molecules were additionally calculated using the MP2 method using the NWChem computational chemistry suite. ${ }^{63}$ The geometry optimization was carried out using the cc-pVDZ basis set. ${ }^{64}$ At the equilibrium structures, we estimated the complete basis set limit of the binding energies using the scheme suggested by Truhlar et al. ${ }^{65}$ The extrapolation was done using the Hartree-Fock and correlation energies obtained with the cc-pVDZ and cc-pVTZ basis sets. The frozen-core approximation was applied in all MP2 calculations.

\section{Results and discussion}

For this study we selected three representative flat molecules of increased size: melamine (M), naphthalene tetracarboxyldianhydride (NTCDA), and PTCDA, all shown in Fig. 1. These particular molecules are widely used in surface studies and were selected, in particular, to see if there is a dependence of the binding energy of the molecules to the gold surface on their size.
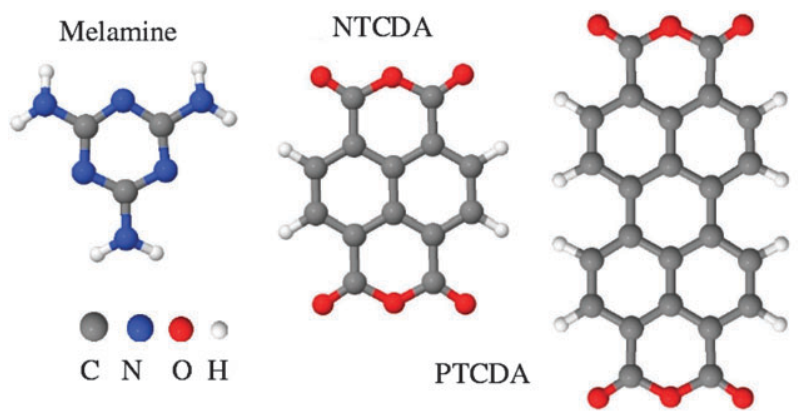

Fig. 1 Melamine, NTCDA and PTCDA molecules.

\subsection{Molecules and dimers}

Firstly, we consistently performed geometry relaxation of the molecules themselves. Note that when doing the vdW-DF calculations, care should be taken of the cell dimension corresponding to the stacking direction of the molecules. We have checked that the total energies of our molecules change by less than $0.01 \mathrm{eV}$ when this cell dimension changes from 20 to $40 \AA$, which is sufficient for our purposes. Our calculations confirmed that both, PBE and vdW-DF methods, produce practically identical results for the bond lengths and bond angles which were found to agree within $0.01 \AA$ and $0.1^{\circ}$, respectively. Then, the relaxation calculations were performed for the corresponding flat $\mathrm{M}-\mathrm{M}$ as well as PTCDA-PTCDA and NTCDA-NTCDA dimers, the latter two were considered in two geometries D1 and D3 as shown in Fig. 2, and, similarly to the single molecules, no major differences were found in the relaxed geometries between the two density functionals.

As far as we are aware, no high-quality quantum chemistry calculations were performed for these dimer systems. Therefore, in order to evaluate the performance of the two density functionals, we also calculated binding energies of the dimers using the MP2 method. As demonstrated by Jurecka et al. ${ }^{66}$ it reliably describes hydrogen-bonded interactions. On the other hand, the benchmark calculations of Goll et al. ${ }^{67}$ imply a decent performance of PBE for hydrogen-bonded complexes. The results of our dimer calculations are presented in Table 1 . The binding energies obtained with the vdW-DF and MP2 are in a reasonable agreement for all systems, however, the PBE energies for NTCDA and PTCDA dimers are too small. This is not surprising since it is well known that hydrogen bonds involving carbon atoms are very weak. What is surprising, however, is that in these cases there is significant contribution of the dispersion interaction to the binding. On the other hand, in the case of the $\mathrm{M}$ dimer bound by strong double $\mathrm{N}-\mathrm{H} \cdots \mathrm{N}$ hydrogen bonds, the dispersion interaction appears to be much less significant. This is also the smallest and therefore the least polarisable complex in our study. The strong binding obtained with PBE for the M dimer is in general accord with previous calculations of strong hydrogen bonded systems ${ }^{56}$ which show an insignificant effect of the dispersion interaction on this type of bonding as compared with the PBE calculations. (a)

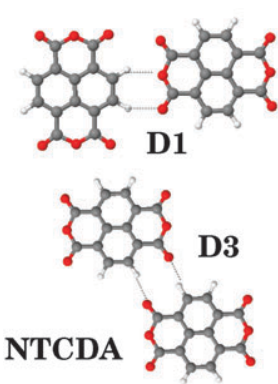

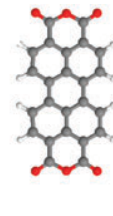

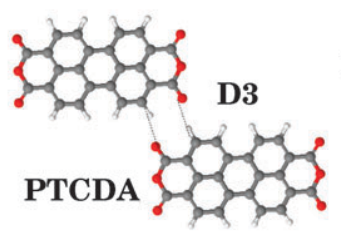

(c)

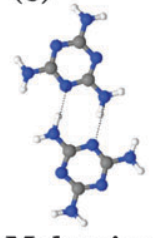

Melamine
Fig. 2 Relaxed geometries of D1 and D3 NTCDA (a) and PTCDA (b) dimers, as well of the $\mathrm{M}$ dimer (c). For convenience, hydrogen bonds are indicated by dashed lines. 
Table 1 Comparison of dimers binding energies (in $\mathrm{eV}$ ) obtained using PBE, MP2 and vdW-DF based calculations. In the cases of PTCDA and NTCDA dimers, two flat configurations D1 and D3 were considered as shown in Fig. 2

\begin{tabular}{llll}
\hline Dimers & PBE & vdW-DF & MP2 \\
\hline M-M & $-0.48^{15}$ & -0.42 & -0.49 \\
NTCDA (D1) & -0.06 & -0.18 & -0.19 \\
NTCDA (D3) & -0.16 & -0.28 & -0.28 \\
PTCDA (D1) & -0.23 & -0.38 & -0.45 \\
PTCDA (D3) & -0.25 & -0.38 & -0.35 \\
\hline
\end{tabular}

Interestingly, the vdW-DF binding energies for the two PTCDA dimers D1 and D3 are found to be the same although our MP2 calculations gave a slight preference (of $0.1 \mathrm{eV}$ ) to the dimer D1. One may think that the vdW-DF calculation in this case fails as it performs qualitatively worse than the MP2 method: the D1 dimer is expected to have stronger both hydrogen bonding and dispersion interactions due to a larger number of "contacts" the two molecules make, and hence the MP2 ordering. We believe, however, that one has to be careful in jumping to conclusions here as both methods are approximate; after all, the energy differences are quite small anyway.

It follows from these results that vdW-DF functional performs extremely well as compared with the MP2 calculations. Not only the order of stability in these systems is correctly reproduced, the absolute values of the binding energies are also very close to the MP2 results. Note in passing that if instead of revPBE we used the PBE exchange in our vdW-DF calculations, a somewhat better agreement with the Coupled Cluster calculations could be expected. ${ }^{57}$ However, we did not exploit this avenue here as it goes beyond the scope of this study.

In the case of the strongly bound $\mathrm{M}$ dimer we have also looked at charge density differences and found a characteristic for the hydrogen bonding "kebab" structure with alternating regions of negative and positive density, which is practically identical to that obtained with the PBE density functional. ${ }^{28}$ Weakly bound NTCDA and PTCDA dimers do not show any well developed "kebab" structure with either of the density functionals. These results may be extended to other molecules which may form hydrogen bonds with each other. Since the stability of two-dimensional assemblies bound by the hydrogen bonding is, for the most part, due to the dimers involved, ${ }^{7,11,12,25,28}$ we can conclude from these calculations that the vdW-DF method gives a description of the lateral interaction between molecules very close to that provided by the GGA functionals, with the exception of weak bonds for which the vdW-DF method provides slightly stronger binding.

\subsection{Molecules and dimers on surfaces}

Bearing in mind the noticeable cost of the calculations due to the large system sizes considered, and the fact that we would mainly like to draw qualitative conclusions in this study on the importance of the dispersion interaction for the moleculesurface systems, all calculations of molecular adsorption on the gold surface were performed using a slab with two layers of gold only, in which the bottom layer atoms were fixed in the bulk geometry, while the upper layer atoms were all free to relax. We carefully checked that using three and four layers of gold (with either one or two layers allowed to relax) changes the binding energies of a melamine (M) molecule on gold only marginally. The lateral dimensions of the unit cells were in all cases chosen such that the interaction between images of adsorbed molecules is negligible: in the case of the adsorption of a single $M$ on gold, a $4 \times 3$ gold cell ( 24 atoms in each layer) was used, while for the $\mathrm{M}$ dimer, NTCDA, and PTCDA adsorption we used a $4 \times 5$ gold cell (40 atoms in each layer). The largest system we considered, a PTCDA on the two-layer gold surface, contained 118 atoms.

Next, we placed single molecules M, NTCDA and PTCDA at random positions in flat geometries on the surface at a distance of around 3.0-3.5 $\AA$ from it. We first discuss our results obtained with the PBE density functional. After the geometry relaxation we find in all cases that the molecules practically remain in the original lateral positions, the only considerable displacement is observed along the direction perpendicular to the surface along which the molecule is displaced as a whole remaining flat. These results hint that the surface potential, as provided by the PBE, must be laterally flat. After the relaxation, the molecules lie flat on the surface at a considerable distance of about $3.5 \AA$, see Table 2 (the experimentally measured distance for PTCDA on the $\mathrm{Au}(111)$ surface is $0.23 \AA$ smaller $\left.{ }^{68}\right)$. The binding energies, shown in the same Table, were found to be very small, of the order of $-0.1 /-0.2 \mathrm{eV}$; moreover, the binding energies of rather large PTCDA and NTCDA molecules have been found to be practically the same as for the small $\mathrm{M}$ molecule. The BSSE corrections in all cases are noticeable and are $0.23,0.24$ and $0.35 \mathrm{eV}$ for the M, NTCDA and PTCDA molecules, respectively. To investigate whether the surface potential is indeed flat, we performed extensive diffusion calculations for the $\mathrm{M}$ and PTCDA molecules on the surface in which a single carbon atom in their benzene-like core was moved in small steps along several directions on the surface with subsequent relaxation of all other atoms of the molecule and the upper layer atoms of the surface; the vertical component of the chosen atom was also allowed to relax. The total displacements for the molecules considered across the surface were up to $3 \AA$. These calculations gave a variation of the binding energy of less than $0.03 \mathrm{eV}$ as a function of the molecules lateral position confirming the fact that the surface potential is indeed extremely flat (see the ESI $\dagger$ ).

The electronic charge density difference for the PTCDA molecule on the gold surface calculated by subtracting the individual electron densities of the molecule and the surface, both calculated in the geometry of the combined system, from the density of the latter, is shown for the PBE calculation in Fig. 3 (upper panel). It clearly demonstrates that there is no density accumulation between the surface and the molecule, although some redistribution of the density within each subsystem is visible. This finding is also supported by the Mulliken population analysis. These conclusions agree with similar calculations of the $\mathrm{M}$ and adenine (A) molecules on the same surface, ${ }^{11,28}$ and are also confirmed by the projected electronic density of states (PDOS) (see discussion below). Thus, according to the PBE based calculations, the binding mechanism for the here considered flat molecules on this metal surface is a weak physisorption. 
Table 2 Interaction of single molecules (of $N$ atoms) with the $\mathrm{Au}(111)$ surface studied using the PBE and vdW-DF methods. In the latter case we show results of both electronically self-consistent (marked "scf") and non-self-consistent (marked "non-scf") calculations. Results of the simulations using the classical potentials ${ }^{61}$ are also given under "Classical" for comparison

\begin{tabular}{|c|c|c|c|c|c|c|c|c|c|}
\hline \multirow[b]{2}{*}{ Molecule } & \multirow[b]{2}{*}{$N$} & \multicolumn{4}{|c|}{ Binding energy/eV } & \multicolumn{4}{|c|}{ Height above the surface $/ \AA$} \\
\hline & & PBE & Non-scf & $\operatorname{scf}$ & Classical & PBE & Non-scf & $\mathrm{scf}$ & Classical \\
\hline $\bar{M}$ & 15 & -0.25 & -0.71 & -0.88 & -1.08 & 3.5 & 3.5 & 3.1 & 2.9 \\
\hline NTCDA & 24 & -0.10 & -1.06 & -1.31 & -1.31 & 3.6 & 3.6 & 3.5 & 3.1 \\
\hline PTCDA & 38 & -0.17 & -1.70 & -1.88 & -2.03 & 3.5 & 3.5 & 3.3 & 3.0 \\
\hline
\end{tabular}
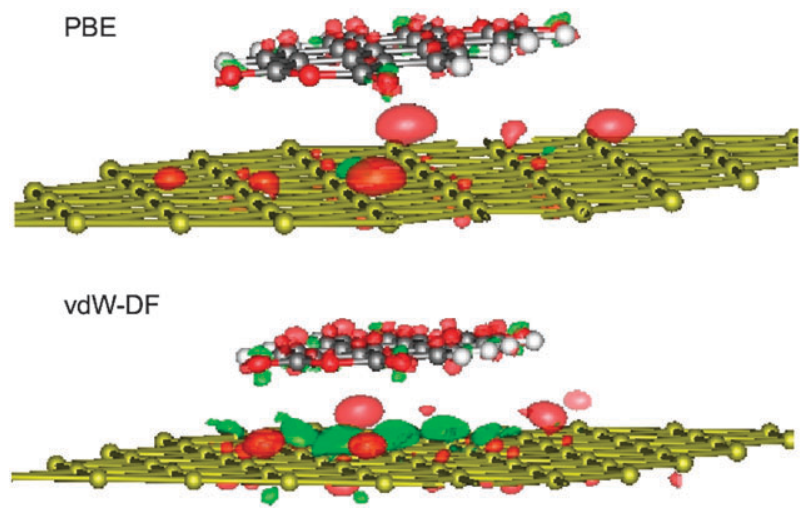

Fig. 3 Electron density difference for the PTCDA molecule on the $\mathrm{Au}(111)$ surface calculated using PBE (top) and vdW-DF (bottom) methods. Red and green contours represent depletion and excess (at $\pm 0.004 \mathrm{e} \AA^{-3}$ ) of electron density, respectively. Only the upper layer of the gold surface is shown.

As anticipated, this picture changes dramatically if the dispersion interaction is accounted for. For comparison, two types of calculations have been performed: non-self-consistent (the PBE electron densities and geometries were used) and self-consistent (fully vdW-DF calculations). The binding energies of the same molecules on the gold surface, calculated self-consistently in the same way as above but using the vdW-DF method and shown in Table 2, become quite significant. Moreover, the binding energy starts to depend on the size of the molecule in accord with the intuitive picture that the vdW interaction is additive: the binding energies of the three molecules relate to each other as $1.4: 1.0: 0.7$ which is close to the corresponding relationship 1.6:1.0:0.6 between the numbers of their atoms. As far as we know, experimental adsorption energies are only available for the NTCDA and PTCDA molecules on gold, ${ }^{69}$ which were measured to be $1.5 \mathrm{eV}$ and $2.0 \mathrm{eV}$, respectively, in a very good agreement with our calculations. Our binding energies are also supported by indirect experimental information on the desorption temperatures of $\mathrm{M}$, naphthalene tetracarboxylic diimide (NTCDI), and perylene tetracarboxylic diimide (PTCDI) molecules from the $\mathrm{Au}(111)$ surface which were found ${ }^{70}$ to be $80{ }^{\circ} \mathrm{C}, 200{ }^{\circ} \mathrm{C}$, and $330{ }^{\circ} \mathrm{C}$, respectively. Note that NTCDI and PTCDI molecules are very similar to the NTCDA and PTCDA molecules considered here, so that desorption temperatures for the latter should be expected to be of the same order of magnitude. Two observations can be made: (i) the vdW-DF binding energies are more consistent with desorption temperatures than the very weak binding predicted by PBE; (ii) there is a clear dependence of the desorption temperatures on the size of the molecules, and, again, the vdW-DF calculations got it right. Of course, one has to be careful in associating the desorption temperatures directly with the adsorption energies of single molecules; however, the trend is only well reproduced by the calculations which account for the dispersion interaction. We note in passing that the BSSE corrections are significant $(0.24 \mathrm{eV}, 0.28 \mathrm{eV}$, and $0.42 \mathrm{eV}$ for the M, NTCDA, and PTCDA, respectively) and are of the same order of magnitude as in the PBE calculations. Due to the cost of the calculations and because we are only interested here in qualitative conclusions, bigger basis set calculations, which would reduce the BSSE, were not tried.

Interestingly, the results for the binding energies obtained in non-self-consistent calculations, as is clear from Table 2, are all systematically lower than those obtained in fully self-consistent calculations. The underestimation of binding varies between $0.17-0.18 \mathrm{eV}$ for $\mathrm{M}$ and PTCDA molecules and $0.25 \mathrm{eV}$ for NTCDA which are of the same order as the total binding energies obtained in our PBE based calculations. These are significant discrepancies: if for PTCDA the error corresponds to only about $10 \%$ of the binding energy, in the cases of M and NTCDA the errors are almost two times larger reaching nearly $20 \%$. These results emphasize the importance of full self-consistency in vdW-DF based calculations.

The geometries of the relaxed systems did not change significantly: the molecules still lie flat above the surface, but at somewhat closer distances than in our PBE calculations (see Table 2). In fact, in the case of PTCDA our calculated distance of $3.3 \AA$ is now much closer to the experimental one of $3.27 \AA .{ }^{68}$ Overall, atomic displacements within the molecules are not larger than $0.01 \AA$. Note that because the revPBE exchange tends to give longer bond lengths, ${ }^{71}$ we may expect that our molecule-to-surface distances are slightly overestimated. The calculated electron densities differences, shown in Fig. 3 (lower panel) for the PTCDA molecule on gold as an example, are very similar to those obtained with the PBE functional, and the previous conclusion of zero charge transfer between the surface and the molecules firmly stays in place in the case of the vdW-DF calculations as well. This result is also confirmed with the PDOS analysis, shown for the PTCDA molecule on gold in Fig. 4. The DOS projected on the PTCDA atoms in the combined system is extremely close to the DOS of an isolated molecule (indicating a small hybridization of the molecular and metal states), and the LUMO (the lowest unoccupied molecular orbital) state of the molecule lies above the Fermi level of the metal, and thus remains unoccupied, suggesting no charge transfer. A similar result was obtained 


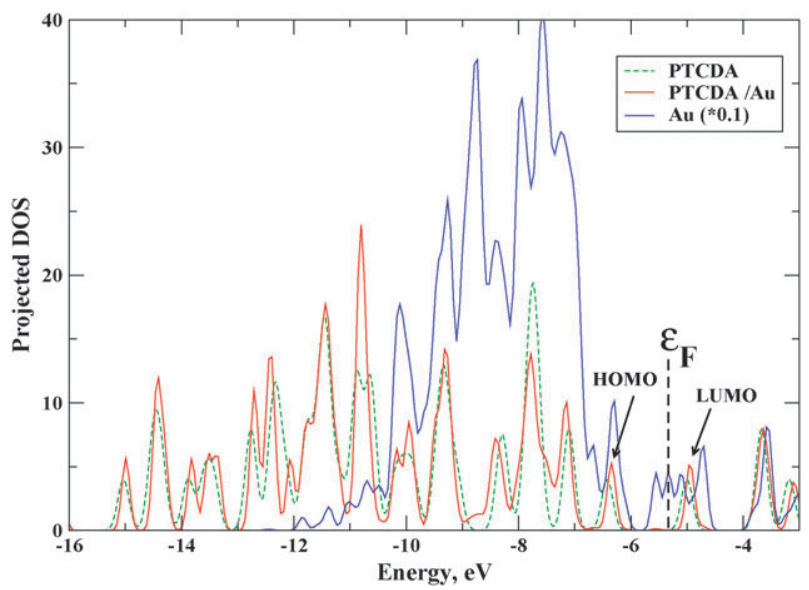

Fig. 4 Electronic projected density of states (PDOS) for the PTCDA molecule on the $\mathrm{Au}(111)$ surface calculated with the vdW-DF method using a single $\Gamma$ point and the Gaussian smearing with $0.2 \mathrm{eV}$ dispersion. The projections are shown on atoms of the molecule (PTCDA/Au, red) and of the surface (Au, blue); the contribution of the surface was reduced 10 times for convenience. The part of the DOS for energies lower than $-16 \mathrm{eV}$ is not shown. The Fermi energy of the metal surface is indicated. For comparison, the total DOS of an isolated PTCDA molecule is also shown by the (green) dashed line with HOMO and LUMO orbitals indicated explicitly.

also in our PBE calculations (not shown). Interestingly, as is seen from Table 2, the binding energies obtained in the vdW-DF based calculations for all three cases considered here are very close to those obtained in classical calculations using the force field developed in ref. 61. However, the force field based calculations predict slightly smaller distances between the molecules and the gold surface.

We have also performed an extensive set of vdW-DF based diffusion calculations for M and PTCDA molecules using the same constrained minimization method as described above for the PBE density functional (see the ESI $\dagger$ ). We find insignificant relaxations of both the gold and the molecules and a very small corrugation of the total energy: when moving the molecules in different lateral directions for up to $3 \AA$, the total energy changes by no more than $0.05 \mathrm{eV}$. This means that the surface potential predicted by the vdW-DF method is also very flat and hence the molecules must be extremely mobile on the surface at small coverages.

These results confirm an intuitive view that, at least for the gold surface studied here, vdW interaction simply provides stronger binding; in fact, this is the main binding mechanism. Otherwise, the electron density of the molecule-gold systems is not significantly modified by the dispersion interaction, and there is no significant change in the electronic DOS as well, as compared with the PBE based calculations.

In order to assess the effect of electronic self-consistency on the electron density when performing vdW-DF calculations, we considered the configuration of the PTCDA molecule on the gold surface relaxed using the vdW-DF method and calculated the electron density of this system using the PBE functional. If a non-self-consistent calculation was performed, this PBE electron density would have been used. The change

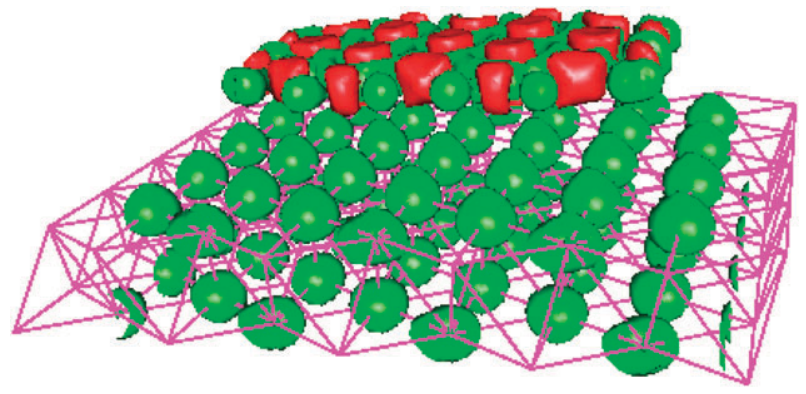

Fig. 5 The change of the electronic density of the PTCDA molecule on the gold surface, calculated using the vdW-DF functional, due to self-consistency. Red and green contours represent negative and

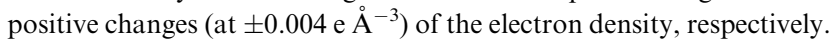

of the electron density due to self-consistency for this system is shown in Fig. 5. Note that the same contour levels are used here as in Fig. 3 which shows electron density difference due to adsorption. The main effect for the gold surface is found to be in some density redistribution around the Au atoms, which is not related to the molecule adsorption. Note that only green contours corresponding to density accumulation around gold atoms are visible as density depletion regions (red) are more localized and hence not visible; the total charge density change within the surface amounts to zero. Further, there is no visible change in the electron density between the molecule and the surface. However, one can notice some accumulation of the density around all atoms of the molecule with subsequent depletion of it in the regions between them, including the regions inside the rings. The absolute values of the density

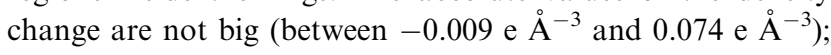
note, however, these are of the same order of magnitude as due to adsorption itself.

Finally, we have considered an $\mathrm{M}$ dimer on the gold using the vdW-DF method. It was shown previously in ref. 28 that a PBE based calculation does not predict any change in the hydrogen bonding between the two molecules due to their (rather weak) interaction with the gold surface, and the overall binding energy of two $\mathrm{M}$ molecules to the gold is basically equal to the sum of the isolated dimer binding energy and twice the single $M$ adsorption energy. Similar results have been obtained here with the vdW-DF method as well; however, the binding to the surface was found to be much stronger. Indeed, the total binding energy of the dimer to the gold surface, calculated with respect to the isolated surface and two separate $\mathrm{M}$ molecules, has been found to be equal to $-2.08 \mathrm{eV}$. If we add the M-M dimer binding energy of $-0.42 \mathrm{eV}$, Table 1 , and twice the adsorption energy for a single $M$ molecule of $-0.88 \mathrm{eV}$, Table 2 , we would get a very similar energy of $-2.18 \mathrm{eV}$. One can also clearly see the well developed "kebab" structure between the two M molecules and the absence of the density in the space between the molecules and the gold in the electron density difference plot for this system in Fig. 6. In fact, the kebab structure is practically unchanged as compared with that for the isolated $\mathrm{M}$ dimer (not shown). This again demonstrates an additivity of the interactions in this system. We observe a very small polarization of the density of the surface, however, no charge transfer to/from the surface is present. 
(a)

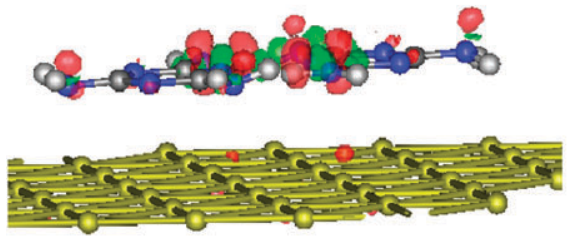

(b)

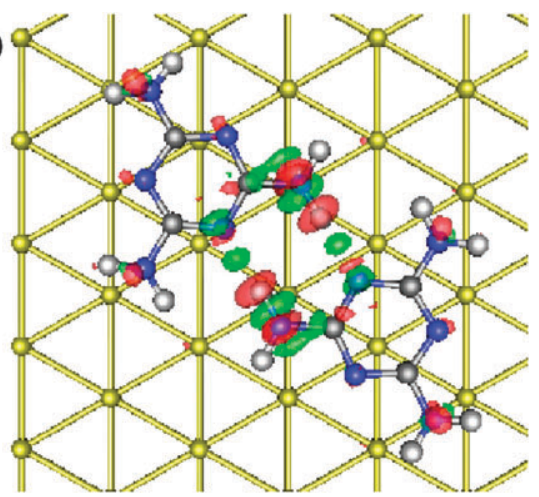

Fig. 6 The side (a) and top (b) views of the electronic density difference plot for the M dimer on the Au(111) surface obtained by subtracting the densities of isolated molecules and of the surface, in the geometries of the combined system, from the density of the latter. The red and green

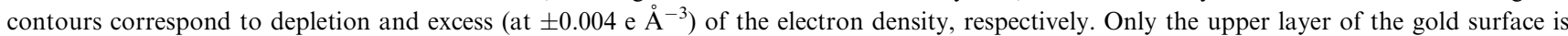
shown.

\section{Conclusions}

We have studied the adsorption of a selection of flat organic molecules of variable sizes on the $\mathrm{Au}(111)$ surface using two density functionals: PBE, which lacks the dispersion interaction, and the vdW-DF, which is completely parameter-free, but accounts for this interaction in an approximate way. All our calculations are fully self-consistent and the vdW interaction was also accounted for in calculating atomic forces. A recently developed ${ }^{57}$ efficient implementation of the vdW-DF method in the SIESTA code ${ }^{60}$ was used.

We find that the vdW interaction affects very little, as compared to the PBE based calculations, the geometries of the molecules on the gold surface. The only significant difference is in that the molecules lie slightly closer to the surface due to vdW interaction. We also find that the vdW-DF method does not effect the molecular systems connected by strong hydrogen bonds; weaker hydrogen bonds may be significantly strengthened by the dispersion interaction. As expected, geometries of these systems remain the same as calculated with the PBE functional, and the order of the dimers with respect to their binding strength does not change either.

However, as anticipated, binding (adsorption) energies of these flat molecules to the $\mathrm{Au}(111)$ surface are affected significantly by the dispersion interaction. While the PBE functional predicts very weak binding which does not depend on the lateral sizes of the molecules, the dispersion interaction as implemented in the vdW-DF method drastically changes this picture: the binding energies increase many times with the dispersion interaction providing most of the binding. We also find that the binding energy depends linearly on the size of these planar molecules, i.e. larger molecules experience larger $\mathrm{vdW}$ interaction to the gold surface as compared to smaller ones. This is in agreement with the available experimental data and intuitive understanding that the $\mathrm{vdW}$ interaction is additive by nature. In addition, we have also considered a $\mathrm{M}-\mathrm{M}$ dimer adsorbed on the gold surface. We find that the vdW interaction does not affect the hydrogen bonding within the dimer. However, as expected, the adsorption energies increase dramatically.

We have also investigated the role of electronic selfconsistency in performing vdW-DF calculations. We found that the change in the electronic density due to self-consistency is of the same order of magnitude as its change for a particular system due to adsorption. Moreover, we find that the binding energies obtained in non-self-consistent calculations are all systematically lower than the ones obtained in fully self-consistent calculations, with the discrepancies reaching in two cases $20 \%$. These results emphasize the importance of performing fully self-consistent calculations of molecules on surfaces, and hence provide an essential justification of the approach adopted here.

For flat surfaces such as the $\mathrm{Au}(111)$, possible twodimensional assemblies are usually constructed and calculated (within DFT) in the gas-phase excluding the molecule-surface interaction and disregarding possible commensurability issues. This is usually justified by the fact that the interaction of the molecules such as melamine, NTCDA, and PTCDA is very weak with this surface, as evidenced by GGA calculations, and that these energies show extremely small corrugation across the surface. This suggests that single molecules must be very mobile during their deposition at room temperature. Since these DFT calculations do not take into account the nonlocal van der Waals contribution to the correlation energy, these results would always remain doubtful unless the $\mathrm{vdW}$ contribution is accounted for. The calculations presented in this paper allow to finally address this point. PBE calculations suggest that molecules on the gold surface can freely move across the surface, however, their evaporation temperatures, due to weak binding, are expected to be very low in strong disagreement with available observations. The $\mathrm{vdW}$ interaction corrects this issue and provides significant binding; the flatness of the surface potential still remains in place, i.e. the molecules can be considered as "floating" in a $2 \mathrm{D}$ pool: they cannot easily escape from the surface, but can freely move around. This is in agreement with the STM observations ${ }^{9,11,12}$ that it is impossible to image small flat molecules on the gold surface at room temperature at small coverages. Our results also imply that the gas-phase modeling, at least in the first approximation, should be adequate for this surface.

These conclusions may also be applied to other metal surfaces, which provide the correct window in the electronic DOS for the molecular states. Namely, one may assume that if 
the metal work function lies between the highest occupied molecular orbital (HOMO) and LUMO of the molecule and there is only a small polarization of the two subsystems (i.e. a small image interaction which otherwise may considerably shift the molecular states), then there will be no charge transfer to/from the molecules and hence the main binding mechanism will be due to dispersion interaction. We believe that this work clearly demonstrates a possibility to perform high quality first-principles DFT calculations with full geometry relaxation on systems containing over hundred of atoms with the dispersion interaction accounted for. Much bigger calculations involving the PTCDA molecule adsorbed on the $\mathrm{KBr}(001)$ surface and containing nearly 250 atoms in the unit cell have been recently performed. ${ }^{58} \mathrm{We}$ hope that this study will stimulate further investigations of the role played by the $\mathrm{vdW}$ interaction in molecule-surface junctions.

\section{Acknowledgements}

M. M. would like to acknowledge the computer time on the HPCx supercomputer via the Materials Chemistry Consortium as well as the financial support from the EPSRC, grant GR/S97521/01. A. G. thanks Academy of Finland for the support through its Centers of Excellence Program (2006-11) and CSC-the IT Center for Science for providing computational resources.

\section{References}

1 J. V. Barth, Annu. Rev. Phys. Chem., 2007, 58, 375-407.

2 A. Kühnle, Curr. Opin. Colloid Interface Sci., 2009, 14, 157.

3 S. De Feyter and F. C. De Schryver, Chem. Soc. Rev., 2003, 32, 139-150.

4 K. Glöckler, C. Seidel, A. Soukopp, M. Sokolowski, E. Umbach, M. Böhringer, R. Berndt and E.-D. Schneider, Surf. Sci., 1998, 405, $1-20$.

5 D. L. Keeling, N. S. Oxtoby, C. Wilson, M. J. Humphry, N. R. Champness and P. H. Beton, Nano Lett., 2003, 3, 9-12.

6 M. Riben, D. Payer, A. Landa, A. Comisso, C. Gattinoni, N. Lin, J.-P. Collin, J.-P. Sauvage, A. De Vita and K. Kern, J. Am. Chem. Soc., 2006, 128, 15644-15651.

7 L. M. A. Perdigão, P. A. Staniec, N. R. Champness, R. E. A. Kelly, L. N. Kantorovich and P. H. Beton, Phys. Rev. B: Condens. Matter Mater. Phys., 2006, 73, 195423.

8 W. Xu, R. E. A. Kelly, M. Schöck, R. Otero, E. Lægsgaard, I. Stensgaard, L. N. Kantorovich and F. Besenbacher, Small, 2007, 3, 2011-2014.

9 R. Otero, M. Lukas, R. E. A. Kelly, W. Xu, E. Lægsgaard, I. Stensgaard, L. N. Kantorovich and F. Besenbacher, Science, 2008, 319, 312-315.

10 T. Classen, M. Lingenfelder, Y. Wang, R. Chopra, C. Virojanadara, U. Starke, G. Constantini, G. Fratesi, S. Fabris, S. de Gironcoli, S. Baroni, S. Har, R. Raval and K. Kern, J. Phys. Chem. A, 2007, 111, 12589-12603.

11 R. E. A. Kelly, W. Xu, M. Lukas, R. Otero, M. Mura, J.-Y. Lee, E. Lægsgaard, I. Stensgaard, L. N. Kantorovich and F. Besenbacher, Small, 2008, 4, 1494-1500.

12 R. E. A. Kelly, M. Lukas, L. N. Kantorovich, R. Otero, W. Xu, M. Mura, E. Laesgaard, I. Stensgaard and F. Besenbacher, J. Chem. Phys., 2008, 129, 184707.

13 S. Wrigelt, J. Schnadt, A. K. Tuxen, F. Masini, C. Bombis, C. Busse, C. an d Isvoranu, E. Ataman, E. Lagsgaard, F. Besenbacher and T. R. Linderoth, J. Am. Chem. Soc., 2008, 130, 5388-5389.

14 R. Otero, W. Xu, M. Lukas, R. E. A. Kelly, E. Laegsgaard, I. Stensgaard, J. Kjems, L. Kantorovich and F. Besenbacher, Angew. Chem., Int. Ed., 2008, 47, 9673-9676.
15 F. Silly, A. Q. Shaw, M. R. Castell, G. A. D. Briggs, M. Mura, N. Martsinovich and L. Kantorovich, J. Phys. Chem. C, 2008, 112, 11476-11480.

16 M. Lukas, R. Kelly, L. Kantorovich, R. Otero, E. Laesgaard, I. Stensgaard and F. Besenbacher, J. Chem. Phys., 2009, 130, 024705 .

17 W. Xu, R. E. A. Kelly, H. Gersen, E. Laesgaard, I. Stensgaard, L. Kantorovich and F. Besenbacher, Small, 2009, 5, 1952-1956.

18 G. Heimel, L. Romaner, J.-L. Brédas and E. Zojer, Phys. Rev. Lett., 2006, 96, 196806.

19 S. Picozzi, A. Pecchia, M. Gheorghe, A. Di Carlo, P. Lugli, B. Delly and M. Elstner, Phys. Rev. B: Condens. Matter Mater. Phys., 2003, 68, 195309.

20 S. X. Du, H. J. Gao, C. Seidel, L. Tsetseris, W. Ji, H. Kopf, L. F. Chi, H. Fuchs, S. J. Pennycook and S. T. Pantelides, Phys. Rev. Lett., 2006, 97, 156105.

21 A. Hauschild, K. Karki, B. C. C. Cowie, M. Rohlfing, F. S. Tautz and M. Sokolowski, Phys. Rev. Lett., 2005, 94, 036106.

22 A. Hauschild, K. Karki, B. C. C. Cowie, M. Rohlfing, F. S. Tautz and M. Sokolowski, Phys. Rev. Lett., 2005, 95, 209602.

23 R. Rurali, L. Lorente and P. Ordejón, Phys. Rev. Lett., 2005, 95, 209601.

24 A. P. Seitsonen, M. Lingenfelder, H. Spillmann, A. Dmitriev, S. Stepanow, N. Lin, K. Kern and J. V. Barth, J. Am. Chem. Soc., 2006, 128, 5634-5635.

25 R. E. A. Kelly and L. N. Kantorovich, Surf. Sci., 2005, 589, $139-152$.

26 A. Bilić, J. R. Reimers, N. S. Hush, R. C. Hoft and M. J. Ford, J. Chem. Theory Comput., 2006, 2, 1093-1105.

27 R. E. A. Kelly and L. N Kantorovich, J. Mater. Chem., 2006, 16, 1894-1905.

28 M. Mura, N. Martsinovich and L. Kantorovich, Nanotechnology, 2008, 19, 465704 .

29 N. Martsinovich and L. Kantorovich, J. Phys. Chem. C, 2008, 112, 17340-17350.

30 L. Romaner, D. Nabok, P. Puschnig, E. Zojer and C. AmbroschDraxl, New J. Phys., 2009, 11, 053010.

31 S. Stepanow, M. Lingenfelder, A. Dmitriev, H. Spillmann, E. Delvigne, N. Lin, X. Deng, C. Cai, J. V. Barth and K. Kern, Nat. Mater., 2004, 3, 229-233.

32 J. V. Barth, G. Costantini and K. Kern, Nature, 2005, 437, 671.

33 M. Stöhr, M. Wahl, C. H. Galka, T. Riehm, T. A. Jung and L. H. Gade, Angew. Chem., Int. Ed., 2005, 44, 7394-7398.

34 L. M. A. Perdigão, E. W. Perkins, J. Ma, P. A. Staniec, B. L. Rogers, N. R. Champness and P. H. Beton, J. Phys. Chem. $B, 2006,110,12539-12542$.

35 F. Silly, A. Q. Shaw, K. Porfyrakis, G. A. D. Briggs and M. R. Castell, Appl. Phys. Lett., 2007, 91, 253109.

36 P. A. Staniec, L. M. A. Perdigão, A. Saywell, N. R. Champness and P. H. Beton, ChemPhysChem, 2007, 8, 2177-2181.

37 R. Madueno, M. T. Räisänen, C. Silien and M. Buck, Nature, 2008, 454, 618-621.

38 A. Saywell, G. Magnano, C. J. Satterley, L. M. A. Perdigão, N. R. Champness, P. H. Beton and J. N. O'Shea, J. Phys. Chem. C, 2008, 112, 7706-7709.

39 C. Silien, M. T. Räisänen and M. Buck, Angew. Chem., Int. Ed., 2009, 48, 3349-3352.

40 Y. Xue, S. Datta and M. A. Ratner, J. Chem. Phys., 2001, 115, 4292-4299.

41 J. Chen, M. A. Reed, A. M. Rawlett and J. M. Tour, Science, 1999, 286, 1550-1552.

42 J. B. Taylor and P. H. Beton, Phys. Rev. Lett., 2006, 97, 236102.

43 U. K. Weber, V. M. Burlakov, L. M. A. Perdigão, R. H J. Fawcett, P. H. Beton, N. R. Champness, J. H. Jefferson, G. A. D. Briggs and D. G. Pettifor, Phys. Rev. Lett., 2008, 100, 156101.

44 F. Silly, U. K. Weber, A. Q. Shaw, V. M. Burlakov, M. R. Castell, G. A. D. Briggs and D. G. Pettifor, Phys. Rev. B: Condens. Matter Mater. Phys., 2008, 77, 201408.

45 J. P. Perdew, K. Burke and M. Ernzerhof, Phys. Rev. Lett., 1996, 77, 3865.

46 S. Priya, P. Puschnig, D. Nabok and C. Ambrosch-Draxl, Phys. Rev. Lett., 2007, 99, 176401.

$47 \mathrm{X} . \mathrm{Wu}, \mathrm{M}$. C. Vargas, S. Nayak, V. Lotrich and G. Scoles, J. Chem. Phys., 2001, 115, 8748. 
48 D. C. Langreth, B. I. Lundqvist, S. D. Chakarova-Kack, V. R. Cooper, M. Dion, P. Hyldgaard, A. Kelkkanen, J. Kleis, Lingzhu Kong, Shen Li, P. G. Moses, E. Murray, A. Puzder, H. Rydberg, E. Schroder and T. Thonhauser, J. Phys.: Condens. Matter, 2009, 21, 084203.

49 S. Grimme, J. Comput. Chem., 2004, 25, 1463-1473.

50 M. Dion, H. Rydberg, E. Schröder, D. C. Langreth and B. I. Lundqvist, Phys. Rev. Lett., 2004, 92, 246401.

51 D. C. Langreth, M. Dion, H. Rydberg, E. Schröder, P. Hyldgaard and B. I. Lundqvist, Int. J. Quantum Chem., 2005, 101, 599-610.

52 T. Thonhauser, V. R. Cooper, S. Li, A. Puzder, P. Hyldgaard and D. C. Langreth, Phys. Rev. B: Condens. Matter Mater. Phys., 2007, 76, 125112.

53 Y. Zhang and W. Yang, Phys. Rev. Lett., 1998, 80, 890.

54 S. D. Chakarova-Käck, E. Schröder, B. I. Lundqvist and D. C. Langreth, Phys. Rev. Lett., 2006, 96, 146107.

55 S. D. Chakarova-Käck, O. Borck, E. Schröder and B. I. Lundqvist, Phys. Rev. B: Condens. Matter Mater. Phys., 2006, 74, 155402.

56 V. R. Cooper, T. Thonhauser, A. Puzder, E. Schröder, B. I. Lundqvist and D. C. Langreth, J. Am. Chem. Soc., 2008, 130, 1304-1308.

57 A. Gulans, M. J. Puska and R. M. Nieminen, Phys. Rev. B: Condens. Matter Mater. Phys., 2009, 79, 201105.

58 O. H. Pakarinen, J. M. Mativetsky, A. Gulans, M. J. Puska, A. S. Foster and P. Grutter, Phys. Rev. B: Condens. Matter Mater. Phys., 2009, 80, 085401.

59 G. Román-Pérez and J. M. Soler, Phys. Rev. Lett., 2009, 103, 096102.

60 J. M. Soler, E. Artacho, J. D. Gale, A. García, J. Junquera, P. Ordejón and D. Sánchez-Portal, J. Phys.: Condens. Matter, 2002, 14, 2745-2779.

61 S. Piana and A. Bilic, J. Phys. Chem. B, 2006, 110, 23467.
62 S. F. Boys and F. Bernardi, Mol. Phys., 1970, 19, 553.

63 E. J. Bylaska, W. A. de Jong, N. Govind, K. Kowalski, T. P. Straatsma, M. Valiev, D. Wang, E. Apra, T. L. Windus, J. Hammond, P. Nichols, S. Hirata, M. T. Hackler, Y. Zhao, P.-D. Fan, R. J. Harrison, M. Dupuis, D. M. A. Smith, J. Nieplocha, V. Tipparaju, M. Krishnan, Q. Wu, T. Van Voorhis, A. A. Auer, M. Nooijen, E. Brown, G. Cisneros, G. I. Fann, H. Fruchtl, J. Garza, K. Hirao, R. Kendall, J. A. Nichols, K. Tsemekhman, K. Wolinski, J. Anchell, D. Bernholdt, P. Borowski, T. Clark, D. Clerc, H. Dachsel, M. Deegan, K. Dyall, D. Elwood, E. Glendening, A. Gutowski, M. Hess, J. Jaffe, B. Johnson, J. Ju, R. Kobayashi, R. Kutteh, Z. Lin, R. Littlefield, X. Long, B. Meng, T. Nakajima, S. Niu, L. Pollack, M. Rosing, G. Sandrone, M. Stave, H. Taylor, G. Thomas, J. van Lenthe, A. Wong and Z. Zhang, NWChem, A Computational Chemistry Package for Parallel Computers, Version 5.1, Pacific Northwest National Laboratory, Richland, Washington, 993520999, USA, 2007.

64 T. H. Dunning, J. Chem. Phys., 1989, 90, 1007-1023.

65 D. G. Truhlar, Chem. Phys. Lett., 1998, 294, 45-48.

66 P. Jureĉka, J. Ŝponer, J. Ĉemý and P. Hobza, Phys. Rev. B: Condens. Matter Mater. Phys., 2009, 79, 201105.

67 E. Goll, T. Leininger, F. R. Manby, A. Mitrushchenkov, H.-J. Werner and H. Stoll, Phys. Chem. Chem. Phys., 2008, 10, 3353-3357.

68 S. K. M. Henze, O. Bauer, T.-L. Lee, M. Sokolowski and F. S. Tautz, Surf. Sci., 2007, 601, 1566-1573.

69 J. Ziroff, P. Gold, A. Bendounan, F. Forster and F. Reinert, Surf. Sci., 2009, 603, 354-358.

70 Wei Xu, private communication, 2009.

71 J. P. Perdew, K. Burke and M. Ernzerhof, Phys. Rev. Lett., 1998, 80, 891. 